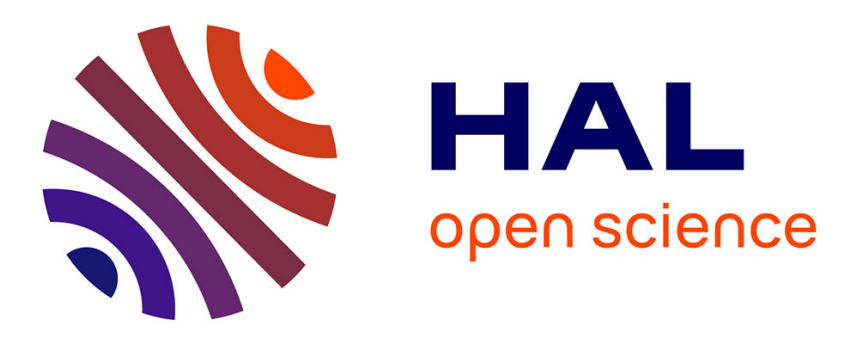

\title{
No-wait Scheduling of a Two-machine Flow-shop to Minimize the Makespan under Non-Availability Constraints and Different Release Dates
}

Faten Ben Chihaoui, Imed Kacem, Atidel B. Hadj-Alouane, Najoua Dridi, Nidhal Rezg

\section{To cite this version:}

Faten Ben Chihaoui, Imed Kacem, Atidel B. Hadj-Alouane, Najoua Dridi, Nidhal Rezg. No-wait Scheduling of a Two-machine Flow-shop to Minimize the Makespan under Non-Availability Constraints and Different Release Dates. International Journal of Production Research, 2011, pp.1. 10.1080/00207543.2010.531775 . hal-00662385

\section{HAL Id: hal-00662385 https://hal.science/hal-00662385}

Submitted on 24 Jan 2012

HAL is a multi-disciplinary open access archive for the deposit and dissemination of scientific research documents, whether they are published or not. The documents may come from teaching and research institutions in France or abroad, or from public or private research centers.
L'archive ouverte pluridisciplinaire HAL, est destinée au dépôt et à la diffusion de documents scientifiques de niveau recherche, publiés ou non, émanant des établissements d'enseignement et de recherche français ou étrangers, des laboratoires publics ou privés. 


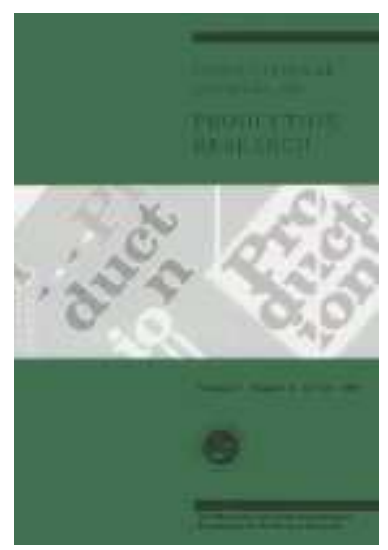

No-wait Scheduling of a Two-machine Flow-shop to Minimize the Makespan under Non-Availability Constraints and Different Release Dates

\begin{tabular}{|c|c|}
\hline Journal: & International Journal of Production Research \\
\hline Manuscript ID: & TPRS-2010-IJPR-0198.R1 \\
\hline Manuscript Type: & Original Manuscript \\
\hline $\begin{array}{l}\text { Date Submitted by the } \\
\text { Author: }\end{array}$ & 07-Aug-2010 \\
\hline Complete List of Authors: & $\begin{array}{l}\text { Ben Chihaoui, Faten; ENIT } \\
\text { Kacem, Imed; Universite Paul Verlaine Metz, Informatique } \\
\text { Hadj-Alouane, Atidel; ENIT } \\
\text { Dridi, Najoua; ENIT } \\
\text { REZG, Nidhal; Universite Paul Verlaine, Metz }\end{array}$ \\
\hline Keywords: & $\begin{array}{l}\text { FLOW SHOP SCHEDULING, AVAILABILITY, MAKESPAN, } \\
\text { MAINTENANCE SCHEDULING, META-HEURISTICS }\end{array}$ \\
\hline Keywords (user): & $\begin{array}{l}\text { scheduling, flow-shop, no-wait, non-availability, branch-and-bound } \\
\text { algorithm }\end{array}$ \\
\hline
\end{tabular}

\section{SCHOLARONE \\ Manuscripts}




\title{
No-wait Scheduling of a Two-machine Flow-shop to Minimize the Makespan under Non-Availability Constraints and Different Release Dates
}

\author{
Faten Ben Chihaoui ${ }^{1}$, Imed Kacem ${ }^{2 *}$, Atidel B. Hadj-Alouane ${ }^{1}$, Najoua Dridi ${ }^{1}$, Nidal Rezg $^{3}$ \\ ${ }^{1}$ OASIS, Ecole Nationale d'Ingénieurs de Tunis, Tunisia \\ ${ }^{2}$ LITA, Université Paul Verlaine Metz, France \\ ${ }^{3}$ LGIPM, COSTEAM INRIA, France
}

\begin{abstract}
In this paper, we consider the two-machine no-wait flow-shop scheduling problem, when every machine is subject to one non-availability constraint and jobs have different release dates. The nonavailability intervals of the machines overlap and they are known in advance. We aim to find a nonresumable schedule that minimizes the makespan. We propose several lower bounds and upper bounds. These bounding procedures are used in a branch-and-bound algorithm. Computational experiments are carried out on a large set of instances and the obtained results show the effectiveness of our method.
\end{abstract}

Keywords: scheduling, flow-shop, no-wait, non-availability, branch-and-bound algorithm.

\section{Introduction}

In this paper, we study the two-machine no-wait flowshop problem under non-availability constraints, when jobs have different release dates. The aim is to minimize the makespan under the three mentioned assumptions. We assume that each machine is unavailable during a fixed interval. The intervals overlap and are known in advance. We assume that if a job cannot be finished before the non-availability period of a machine, the job needs to completely restart once the machine becomes available. This practical assumption is motivated by several real practical situations (preventive maintenance for instance). The second practical assumption considered in this work is related to the no-wait constraint. The main reasons of such a constraint consist in the technological

\footnotetext{
*kacem@univ-metz.fr (Corresponding author)
} 
structure of the shop itself. In no-wait scheduling, a job has to be continuously processed without idle-time between successive machines. Given the aim of this study, we recall some works related to the considered application and assumptions.

The numerous applications of our first assumption can be found in the paper by Bagchi et al (2006), in which they proposed several no-wait and blocking scheduling models. Moreover, they illustrated some ways in which the used modern manufacturing systems such as robotic cells may be modelled as a TSP (Travelling Salesman problem). Ronconi (2005) considered the minimization of the makespan criterion for the flowshop problem with blocking. A lower bound exploiting the occurrence of blocking is proposed. A branch-and-bound algorithm incorporating this lower bound is described and its efficiency is evaluated on several problem instances. The makespan minimization problem in a two-machine flowshop under no-wait constraints can be solved to optimality in $O(n \log n)$ time, where $n$ is the number of jobs (Gilmore and Gomory 1964). However, this problem is strongly NP-hard for $m \geq 3$ where $m$ is the number of machines, even if the buffer storage is limited (Röck, 1980). Moreover, if the no-wait constraint is restricted to a sub-set of jobs then, the problem remains NP-hard in the strong sense (Finke et al 1997). The $m$-machine no-wait flowshop scheduling problem with the aim of minimizing the makespan and the total completion time was studied in Allahverdi and Aldowaisan (2002). A dominance rule and heuristics were proposed and used in a branch-and-bound algorithm. For more details on no-wait and blocking scheduling problems, the reader is invited to consult the state-of-the-art paper by Hall and Sriskandarajah (1996).

The second assumption, that is, the non-availability constraint is one of the new modern concepts in the scheduling theory. Abundant literature exists on the related problems. Lee was the pioneer of this research field in scheduling theory (Lee 1997). Many papers were published during the last two decades and they involved various works on the flow-shop configuration. The makespan minimization on the two-machine flow-shop problem under non-availability constraints was proven to be NP-hard, even with a single non-availability period (Espinouse et al 1999). Aggoune and Portmann proposed a heuristic method for the general flow-shop problem under non-availability constraints on a subset of the machines (Aggoune and Portmann 2006). Lee studied the twomachine flowshop problem under the assumption that the non-availability time is known in advance (Lee 1999). Moreover, he considered the semiresumable, the resumable and the nonresumable cases. Lee also conducted a complexity analysis and elaborated a pseudo-polynomial dynamic programming algorithm to solve the problem to optimality. Heuristic algorithms were proposed and evaluated in the worst-case (Lee 1999). Allaoui et al. considered the same problem with a single 
non-availability interval on the first machine under the nonresumable scenario (Allaoui et al 2006). They improved the dynamic programming model proposed by Lee (1997). This method allowed them to reduce the computational effort. Some conditions, where Johnson's rule gives the optimal solution were specified. They proved that the worst-case performance bound of Johnson's rule is 2 (Allaoui et al 2006). For more details on the non-availability constraints in scheduling problems, we refer to the survey-papers by Schmidt (2000) and Ma et al. (2010).

Finally, our third assumption states that jobs have different release dates. Several literature works considered this case. A sample of them is summarized as follows. First, we note that the classical version consisting in the makespan minimization on two-machine flow-shop subject to jobs release dates $\left(F 2 / r_{j} / C_{\max }\right)$ is $N P$-hard in the strong sense (Lenstra et al 1977). Thus, heuristic approaches were widely studied. In (Potts 1985) four heuristics were proposed to solve the same problem. For three of them, the worst-case performance ratio is of 2. Each one of the heuristics can be implemented in $O(n \log n)$ time. The fourth one is based on the iterative use of the third heuristic and it has a worst-case performance ratio of $5 / 3$ and a time complexity of $O\left(n^{3} \log n\right)$. In (Kashyrskikh 2001), by modifying Potts algorithm (Potts 1985), the authors reduced the worst-case performance ratio to $3 / 2$ however, the time complexity remains in $O\left(n^{3} \log n\right)$. A polynomial time approximation scheme (PTAS) was proposed in (Hall 1995). In (Kovalyov and Werner 1997) a polynomial approximation procedure was elaborated for the $F 2 / r_{j} / C_{\max }$ problem. Such a procedure was based on a dynamic programming approach using modified release dates and processing times. Compared to the one proposed in (Hall 1994) a better time complexity was obtained for large values of $n$. Branch-and-bound methods were exploited in (Cheng et al 2001) for solving the $F 3 / r_{j} / C_{\max }$ problem by incorporating a lower bound determined for some particular cases. Two dominance rules were used to construct an initial schedule. These rules reduced the search space by decomposing the problem into a finite set of sub-problems.

To conclude, according to this literature review we can mention that in all studied papers, at most two of the three assumptions were considered. A sample of these works includes Cheng and Liu 2003, Espinouse et al 1999, Espinouse et al 2001, Kubzin and Strusevich 2004 and Wang and Cheng 2001, where no-wait and non-availability constraints were jointly considered. Finally, the non-availability constraint and the different job release dates were considered at the same time by França et al 2006, where genetic algorithms were elaborated. Moreover, the same problem was solved in Bianco et al 1999 by a mathematical programming method and two heuristics. However, according to the best of our knowledge there is no previous work related to the studied problem, 
that is, the minimization of the makespan in two-machine no-wait flow-shop under non-availability constraints and different job release dates assumption. For this reason, this paper is a first successful attempt to design a branch-and-bound algorithm with an interesting performance.

The reminder of the paper is organized as follows. Section 2 gives a precise formulation. In Section 3, we describe the proposed branch-and-bound method. Computational results are given and discussed in Section 4. Finally, we conclude the paper by some remarks and perspectives in the last section.

\section{Problem formulation}

The problem can be stated as follows. We have a set $J=\{1, \ldots, n\}$ of $n$ jobs to be performed on two machines $M_{1}$ and $M_{2}$. Every job has to be processed first on $M_{1}$ then on $M_{2}$. For every job, the second operation has to start immediately at the end of the first operation (no idle-time is allowed between two consecutive operations of a given job). Every machine $M_{i}(i=1,2)$ is unavailable during the interval $\left(s_{i}, t_{i}\right)$. Due to practical requirements we assume that the two intervals $\left(s_{1}, t_{1}\right)$ and $\left(s_{2}, t_{2}\right)$ overlap and that $s_{1} \leq s_{2}$ and $t_{1} \leq t_{2}$. Jobs have to be processed under the nonresumable scenario (a job has to completely restart once interrupted by a non-availability interval). Every job $j$ $(j=1, \ldots, n)$ has a positive release date $r_{j}$ known in advance. Its first (respectively the second) operation on the first (respectively the second) machine has a positive processing time of $a_{j}$ (respectively of $b_{j}$ ). The objective is to find a feasible schedule with the aim of minimizing the makespan (i.e., the completion time of the last operation performed on the second machine). From Section 1, this problem is NP-hard in the strong sense since it is a generalization of other problems of this type (for instance, see the $F 2 / r_{j} / C_{\max }$ problem in Lenstra et al 1977).

\section{Branch-and-bound algorithm}

Motivated by the practical advantages of the branch-and-bound approach, we elaborated an algorithm of this type to solve our considered problem. Such an approach aims to find an optimal solution (or an enhanced one) by reducing the search space based on different tools (lower bounds, dominance rules, upper bounds...). When it is not possible to obtain the optimal solution such an approach allows us to improve the result of other heuristic methods that can be used as an initial upper bound. In this section we give the description of our branch-and-bound algorithm. It will be noted $\mathrm{B} \& \mathrm{~B}$ in the remainder of the paper. 


\subsection{Branching scheme and search strategy}

The B\&B starts by computing an initial solution which provides the first upper bound. Every node represents a partial schedule. The branching scheme consists in scheduling a new job after a partial schedule. The search space is explored by using the depth first strategy. Before creating a new node, a lower bound is computed. If the value of such a lower bound is greater than the value of the upper bound, then this node is removed. Moreover, before any branching procedure, we increase the release date of every jobs $j \in J$ satisfying one of the two following conditions: $\left(r_{j}+a_{j}>s_{1}\right)$ or $\left(r_{j}+a_{j}+b_{j}>s_{2}\right)$. Indeed, in the two cases the first operation of job $j$ cannot be performed before $s_{1}$. Thus, we can increase the release date as follows: $r_{j}=\max \left\{r_{j}, t_{1}, t_{2}-a_{j}\right\}$. Consequently, all the partial sequences beginning with these jobs verifying one of the conditions are eliminated.

\subsection{Upper bounds}

The quality of the used upper bounds is very important to enhance the effectiveness of any branchand-bound algorithm. For this reason, we investigated different ways to determine the initial upper bound. Three methods are used:

- A greedy search (denoted as $G S$ ): it consists in applying the $\mathrm{B} \& \mathrm{~B}$ for only $n$ nodes by using the depth best first strategy.

- Two Heuristics (H1 and H2) developed by Ben Chihaoui et al 2009.

- A genetic algorithm (denoted as $G A)$.

For self-consistency, we recall the principle of of Heuristics (H1 and H2). These two heuristics are based on the algorithms in Gilmore-Gomory 1964 and Cheng and Liu 2003. Before describing these heuristics, we present additional notations.

- $I_{k}$ : Set of jobs arriving at the time $r_{k}$;

- $O_{k}\left(D_{k}\right)$ : Set of jobs of the sub-problem $k$ in $H 1(H 2)$;

- $\sigma_{k}^{1}\left(\sigma_{k}^{2}\right)$ : The sequence found by scheduling the jobs of $O_{k}\left(D_{k}\right)$ in $H 1(H 2)$;

- $C L$ : Algorithm 3 of Cheng and Liu 2003.

\section{Description of $C L$}

In Cheng and Liu 2003, the authors study the two-machine no-wait flowshop problem in which each machine may have an unavailable interval. A 3/2-approximation algorithm is developed for the problem resolution when the unavailable intervals on the two machines overlap. It consists in the following steps: 
1. Try to find a good schedule in which the availability constraint is inactive (a fictitious job is added).

2. Relax the availability constraint, and then move some jobs from the beginning to the end or vice versa to meet the unavailability constraint.

3. Optimally schedule some critical job and its adjacent jobs and schedule the other jobs according to Gilmore and Gomory's algorithm.

\section{Description of $\mathrm{HI}$}

The first heuristic, $H 1$, is based on the decomposition of the problem into many sub-problems. The first sub-problem deals with the set $I_{0}$ of jobs that are available at the date $r_{0}$. The (CL) algorithm is used for its resolution. From the obtained solution, we keep the sequence $\sigma_{0}^{1}$ of jobs starting their execution on $M_{1}$, before the date $r_{1}$. The next sub-problem concerns jobs of the set $O_{1}$, which includes jobs that are in $I_{1}$ but not in $\sigma_{0}^{1}$. The solution obtained for this sub-problem is concatenated to $\sigma_{0}^{1}$ to obtain a new partial sequence. Then, we consider as many sub-problems as the number of release dates $r_{k}$.

\section{Description of $\mathrm{H2}$}

The second heuristic, $H 2$, is based on a modification of $H 1$ to use the concept of a reactive scheduling. Indeed, a job $j$ can arrive to the shop after a job $i$. However, the execution of $j$ before $i$ may give a better solution.

In $H 2$, sub-problems are defined as follows. First, jobs arriving at $r_{i}$ are temporarily scheduled to obtain their finish date, referred to as $f_{i}$. Then the sub-problem consists in rescheduling all jobs arriving at $r_{i}$ along with jobs arriving before $f_{i}$.

The first sub-problems concern the jobs in set $D_{0}$. We apply $C L$ to schedule set $I_{0}$. The makespan obtained is considered as $f_{0}$. Set $D_{0}$ includes jobs of set $I_{0}$ and those which release dates verify the condition: $r_{0} \leq r_{j} \leq f_{0}$. Let $r_{k}=\max _{j}\left\{r_{j}\right\}$. We keep from the solution obtained by the application of $C L$ on $D_{0}$, the sequence of jobs beginning their execution on $M_{1}$ before the date $r_{k+1}$. Let $\sigma_{k}^{2}$ be the obtained sequence.

The second sub-problem concerns jobs of set $D_{1}$.

$C L$ is applied to schedule the set $I_{k+1}$ to which we include jobs of the set $D_{0}$ not kept in the sequence $\sigma_{0}^{2}$. The makespan of the resulted solution is $f_{1}$. Beside the jobs of the set $I_{k+1}, D_{1}$ includes 
jobs arriving after $r_{k+1}$ in the time period of $f_{1}$. The solution obtained for this sub-problem is concatenated to $\sigma_{0}^{2}$ to obtain a new partial sequence (Ben Chihaoui et al 2009).

\section{Description of the genetic algorithm:}

We use a classical genetic algorithm in which the solutions or chromosomes are represented as a permutation of $J$ indicating for every job its position in the schedule (Fig.1 (a)). The mutation operator consists in choosing two random positions of the mutated genes and in swapping the two corresponding jobs (Fig.1 (b)). The crossover operator consists in selecting a random position and in exchanging the genetic information between two parents to construct two offspring according to the selected position (Fig.1 (c)). The construction consists in copying each subset of jobs in the same order as they appear in the corresponding parent so that the offspring will be feasible. The number of generations is fixed to 100 and the initial population is fixed to 100 chromosomes randomly generated by iteratively mutating the FIFO sequence (the FIFO sequence consists in scheduling jobs in non-decreasing order of their release dates).

The initial upper bound used in the $B \& B$ is the minimum of the obtained values with these three methods.

\section{//Please insert Figure 1 about here}

\subsection{Lower bounds}

It is well known that the quality of the lower bound is one of the most critical elements of any branch-and-bound algorithm. In most cases, computing a lower bound consists in relaxing some constraints (in different ways) and in solving a new easier problem. In this paper, we consider two main relaxations to derive lower bounds. The first one replaces the two non-availability intervals by two fictitious jobs. The second relaxation reduces the flow-shop configuration to a single-machine problem. Before presenting the proposed lower bounds we need to define the data of the fictitious jobs. Let $f 1$ be the first fictitious job and $f 2$ be the second one. Their processing times are defined as follows: $a_{f 1}=s_{2}-s_{1}, b_{f 1}=t_{1}-s_{2}, r_{f 1}=s_{1}, a_{f 2}=t_{1}-s_{2}, b_{f 2}=t_{2}-t_{1}, r_{f 2}=s_{2}$. Moreover, we define a global set of jobs $J_{f}$ as follows $J_{f}=J \cup\{f 1, f 2\}$.

We derived five lower bounds denoted: $L B 1, L B 2, L B 3, L B 4$ and $L B 5$. Before computing each lower bound the release date of every job $j \in J$, not yet scheduled, is updated according to whether or not the completion time of the scheduled jobs is greater than the starting time of the non-availability interval on machine $M_{l}$. The construction of every lower bound is described as follows. 


\subsection{1. $L B 1$}

In this lower bound, we transform the problem into an instance of a single-machine problem of the type $1 / r_{j} / \mathrm{C}_{\max }$. More precisely, the first machine is removed and we only consider the second machine. In the new obtained instance, we first generate the two fictitious jobs. Then, with every $j \in J_{f}$ it is associated a new job to be performed on the single-machine such that the release date and the processing time are respectively equal to $r_{j}+a_{j}$ and $b_{j}$. The resulting problem is optimally solved by the FIFO (First In First Out) rule.

\subsection{2. $L B 2$}

In this lower bound, we transform the problem into an instance of the type $1, h_{1} / r_{j}, q_{j} / C_{\max }$. More precisely, the second machine is removed and the second operation of every job $j \in J_{f}$ is replaced by a tail (or a delivery time) equal to $b_{j}$. In the new obtained instance, we first generate the two fictitious jobs. Then, with every $j \in J_{f}$ it is associated a new job to be performed on the singlemachine such that the release date, the processing time and the tail are respectively equal to $r_{j}, a_{j}$ and $b_{j}$. Moreover, the new fictitious jobs must start exactly at their respective release dates. The resulting problem is NP-Hard, but the lower bound is obtained by solving the preemptive version to optimality using Jackson's rule (see Carlier et al 2010).

\subsection{3. $L B 3$}

The principle of this bound is similar to the previous one. We follow the same relaxations as in LB2 to transform the problem into an instance of the type $1 / r_{j}, q_{j} / C_{\max }$. The only difference is the fact that the new jobs associated with the fictitious jobs are not constrained to start exactly at their respective release dates. The resulting problem is solved by the branch-and-bound proposed in Carlier (1982).

\subsection{4. $L B 4$}

The principle of this bound is based on the relaxations used in $L B 1$. We follow the same relaxations. The only difference is that the new jobs associated with the fictitious jobs are constrained to be performed exactly in the interval $\left(s_{2}, t_{2}\right)$. Thus, they can be considered as a non-availability interval. Moreover, the resumable scenario is considered. The resulting problem belongs to the $1, h_{1} / r_{j}, r s / C_{\max }$ family and it is solved to optimality by the preemptive FIFO rule. Note that an analytical comparison can easily show that $L B 4$ gives the same result as $L B 1$. Hence, this bound will be omitted. However, we felt it is very important to report it to show that this idea is not more 
productive than $L B 1$.

\subsubsection{LB5}

The fifth lower bound is obtained by transforming the problem into an instance of the $1, h_{1} / r_{j}$, $n r s / C_{\max }$ type. In such a case, the first machine is removed and we only consider the second machine. In the new obtained instance, we do not generate any fictitious job. Then, with every $j \in J$ it is associated a new job to be performed on the single-machine such that the release date and the processing time are respectively equal to $r_{j}+a_{j}$ and $b_{j}$. Moreover, the single-machine is considered as non-available during the interval $\left(s_{2}, t_{2}\right)$. The resulting problem is solved (under the nonresumable scenario) by the dynamic programming algorithm proposed in Kacem and Haouari (2009). Clearly, this bound outperforms LB1. However, it needs more computation time.

\section{Numerical experiments}

In this section, we describe the numerical experiments carried out in order to evaluate our algorithms. The B\&B was implemented in the $\mathrm{C}$ language and tested on an Intel Pentium IV $3 \mathrm{GHz}$ processor and $512 \mathrm{M}$ RAM, in the WINDOWS XP environment. The instances were randomly generated. For the experiments, we generated ten instances for every combination of parameters as follows. The number of jobs $n$ was chosen in $\{5,10,15,20\}$. The release dates were uniformly distributed in the interval $[1,100 * R]$, where $R \in\{1,2, n, 2 n\}$. Processing times $a_{j}$ and $b_{j}$ were uniformly distributed in the interval $[1,100]$. The horizon $T$ of the schedule is defined as the sum of all the processing times $T=\sum_{j \in J} a_{j}+\sum_{j \in J} b_{j}$. Then, the non-availability periods were fixed at the beginning $\left(s_{1}=0.25 \times T\right)$ or in the middle $\left(s_{1}=0.5 \times T\right)$ of the defined horizon, where $t_{1}=s_{1}+T / n, s_{2}=s_{1}+0.25 \times T / n$ and $t_{2}=s_{2}+1.25 \times T / n$. Based on the values of parameters $R$ and $s_{1}$, we define eight groups of instances $(1,2,3,4,5,6,7$ and 8$)$ as it is shown in Table 1.

\section{//Please insert Table 1 about here}

\subsection{Lower and upper bounds evaluation}

To evaluate the performance of the lower bounds presented in this paper, we compute the average value obtained from ten instances for every combination of parameters. In order to check the effectiveness of $G S$ and $G A$ compared with $H 1$ and $H 2$, we compute the average value obtained from the ten instances. The different results are reported in Tables 2-6. 
For every group the best lower bound (LB) is written in boldface. For the upper bound (UB) the Gap in percentage is calculated with respect to the best lower bound.

\section{$\underline{\text { //Please insert Table } 2 \text { about here }}$ \\ //Please insert Table 3 about here //Please insert Table 4 about here \\ //Please insert Table 5 about here \\ //Please insert Table 6 about here}

From Tables 2-6, we can make the following remarks:

- Except for the case where $n=5$, one can remark that either $G S$ or $G A$ gives the best results in most cases.

- For the lower ranges of release dates, $H 2$ gives better results than $H 1$. This is due to the reactive aspect of $H 2$. However, for a larger range of release dates, $H 1$ gives better results than $H 2$.

- For the lower range of the release dates, LB5 is the best lower bound. However, we can remark that $L B 1$ gives almost the same results as $L B 5$. Hence, $L B 1$ represents an interesting bound with a good quality and a short computation time.

- For a larger range of the release dates values, the different lower bounds give similar results.

- When the non-availability is in the middle of the horizon, the bounds values are generally better (the gap is smaller), and we notice that $L B 5$ and $L B 1$ generally remain the best in the two cases.

- The computation time of the different lower bounds is close to zero. However, the computation time of $L B 5$ for $n=20$ is about $120 s$.

- The gap between the lower and upper bounds values is relatively small. This allows us to elaborate an efficient B\&B.

Given the above remarks, and taking into account the time complexity of every lower bound, we have decided to mainly use $L B 1$ in the B\&B and to employ LB3 and LB5 in the Greedy Search.

\section{2. $B \& B$ performance}

In order to evaluate the $\mathrm{B} \& \mathrm{~B}$ performance, computational experiments are conducted on the same instances used to evaluate the LB and UB. The computation time is limited to $7200 s$. For the node evaluation we use first $L B 1$, if the node is not eliminated, we use $L B 5$. The $\mathrm{B} \& \mathrm{~B}$ performances are 
presented in Tables 7-10, where the following parameters are reported:

- Copt: average of the optimal makespan values

- $U B$ : average of the best upper bound values

- $C P U$ : average of the $\mathrm{B} \& \mathrm{~B}$ computation time values in seconds

- $\quad N_{d}$ : average number of eliminated nodes in the preliminary elimination

- $N_{e x}$ : average number of explored nodes

- $\quad N_{e l}$ : average number of eliminated nodes

- $G a p(\%)$ : average value of the gap between $U B$ and Copt

\section{//Please insert Table 7 about here}

//Please insert Table 8 about here

//Please insert Table 9 about here

\section{//Please insert Table 10 about here}

From Tables 7-10, we can make the following remarks:

- The average value of the gap is about $3 \%$. This value clearly shows the good performance of the $\mathrm{B} \& \mathrm{~B}$ and the tightness of the upper and lower bounds.

- The average value of the gap decreases when $n$ increases.

- Computation time is larger for higher values of $n$.

- Computation time and average number of the explored nodes are relatively high when the release dates range is independent of $n\left(r_{j}\right.$ in [1,100] and in [1,200]) and the non-availability interval is in the middle of the horizon. Consequently, the problem is more difficult for lower range of release dates and when the non-availability is in the middle of the horizon. Indeed, if the non-availability is in the beginning of the horizon, it is skipped early in the horizon (after sequencing some jobs), then the two machines become available.

- The B\&B can solve instances with up to 20 jobs, except for instances of groups 1 to 4 ( $r_{j}$ in $[1,100]$ and in $[1,200])$. Indeed, the low range of release dates in these groups increases the search space. Consequently, the search tree is bigger and the problem is difficult to solve.

- The preliminary elimination is more efficient when $n$ is large.

- The lower bounds and, in particular, LB1 and LB5 allow for an extensive elimination of nodes. 
Thus, they enhance the B\&B effectiveness.

For larger values of $n$ (30, 40 and 50) we tested the performance of the Greedy Search heuristic by reporting the following parameters in Table 8:

- $L B$ : average of the best lower bound values

- $t \_G S$ : average of the $G S$ computation times

- $\quad G \_H(\%)$ : average value of the gap between $H(H$ can be $H 1, H 2, G A$ and $G S)$ and LB.

The average values of the computation times required by $H 1, H 2$ and $G A$ are close to zero second. The obtained performances are reported in Table 11.

\section{//Please insert Table 11 about here}

From Table 11, we can make the following conclusions:

- The maximum value of the gap obtained for the different methods is in average of $11 \%$ and the minimum is of $1 \%$. Thus, the methods that we developed as well as the elaborated lower bounds yield good results.

- The value of $G \_G S$ is of $1 \%$ whereas $G_{-} G A \approx 8 \%, G \_H 1 \approx 4 \%$ and $G_{-} H 2 \approx 11 \%$. Hence, the Greedy Search clearly outperforms all the other algorithms. The GS has a larger computation time (about $0,4 \mathrm{~s}$ in average) than the other algorithms. Nevertheless, such a value is very small in practical situations.

- In conclusion, our $G S$ algorithm seems to be a very interesting method in order to obtain a nearoptimal solution (gap of $1 \%$ ) in a short computation time (in less than $1 s$ ) despite the strong $N P$ Hardness of the studied problem.

\section{Conclusion}

This paper studied the two-machine no-wait flow-shop scheduling problem, when every machine is subject to one non-availability constraint and jobs have different release dates. The aim is to minimize the makespan. Several lower and upper bounds are proposed and incorporated in a branch-and-bound algorithm. Numerical experiments were carried out on a large set of instances. The obtained results showed that we can find the optimal solution for problems with up to 20 jobs within a reasonable amount of computation time. Moreover, the branch-and-bound algorithm can be converted into a greedy search heuristic, GS, that has a good performance. Such a heuristic is able to give a near-optimal solution (gap of $1 \%$ ) in a short computation time (in less than $1 s$ ) despite the 


\section{References}

Aggoune, R., Portmann, M.C (2006). Flow shop scheduling problem with limited machine availability: A heuristic approach. International Journal of Production Economics 99, 4-16.

Allaoui, A., Artiba, A., Elmaghraby, S.E., Riane, F (2006). Scheduling of a two-machine flowshop with availability constraints on the first machine. International Journal of Production Economics 99, 16-27.

Allahverdi, A., Aldowaisan, T. (2002). No-Wait Flowshops with Bicriteria of Makespan and Total Completion Time. Journal of the Operational Research Society 53:9, 1004-1015.

Bagchi, T.P., Gupta, J.N.D., Sriskandarajah, C (2006). A review of TSP based approaches for flowshop scheduling. European Journal of Operational Research 169, 816-854.

Bianco, L., Dell'Olmo, P., Giordani, S (1999). Flow shop no-wait scheduling with sequence dependent setup times and release dates. INFOR 37:1, 3-19.

Carlier, J (1982). The one-machine sequencing problem. European Journal of Operational Research 11, 42-47.

Carlier, J., Hermès, F., Moukrim, A., and Ghédira, K (2010). Exact resolution of the one-machine sequencing problem with no machine idle time. Computers \& Industrial Engineering DOI:10.1016/j.cie.2010.03.007.

Cheng, J., Steiner, G., Stephenson, P (2001). A computational study with a new algorithm for threemachine permutation flow shop problem with release times. European Journal of Operational Research 130, 559-575.

Cheng, T.C. E., Liu, Z (2003). 3/2 approximation for two-machine no-wait flowshop scheduling with availability constraints. Information Processing Letters 88, 161-165.

Ben Chihaoui, F., Dridi, N., Ben Hadj-Alouane, A (2009). Two-machine No-wait Flowshop Scheduling with Availability Constraints and Release Dates. Proceedings of the 2009 International Conference on Computers \& Industrial Engineering, 6-8 july 2009, Troyes, 
France.

Espinouse, M.L., Formanowicz, P., Penz, B (1999). Minimizing the makespan in the two-machine no-wait flow-shop with limited machine availability. Computers \& Industrial Engineering 37, 497-500.

Espinouse, M.L., Formanowicz, P., Penz, B (2001). Complexity results and approximation for the two-machine no-wait flow-shop with limited machine availability. Journal of the Operational Research Society 52, 116-121.

Finke, G., Espinouse, M.L., Jiang, H (1997). Flowshops and extensions. In: Proceedings: Conference on Management and Control of Production and Logistics (MCPL'97), Campinas, Brazil, 275-280.

França, P. M., Tin, G., Buriol, L.S (2006). Genetic algorithms for the no-wait flowshop sequencing problem with time restrictions. International Journal of Production Research 44, 939-957.

Gilmore, P.C., Gomory, R.E (1964). Sequencing a one-state variable machine: a solvable case of the travelling salesman problem. Operations Research 12, 655-679.

Hall, L.A (1994). A polynomial approximation scheme for a constrained flow-shop scheduling problem. Mathematics of Operations Research 19, 68-85.

Hall, L.A (1995). Approximability of flowshop scheduling. Proceedings of the 36th Annual Symposium on Foundations of Computer Science, IEEE Comput. Soc. press, Los Alamitos, 82-91.

Hall, N.G., Sriskandarajah, C (1996). A survey of machine scheduling problems with blocking and no-wait in process. Operations Research 44:3, 510-525.

Kacem, I., M. Haouari, M (2009). Approximation algorithms for single machine scheduling with one unavailability period. 4OR: A Quarterly Journal of Operations Research 7:1, 79-92.

Kashyrskikh, K.N., Potts, C.N., Sevastianov, S.V (2001). A (3/2)-approximation algorithm for twomachine flwo-shop sequencing subject to release dates. Discrete Applied Mathematics 114, 255-271.

Kubzin, M.A., Strusevich, V.A (2004). Approximation Algorithms for Two-Machine Flow Shop NoWait Scheduling with a Non-Availability Interval. Naval Research Logistics 51:4, 613 - 631.

Kovalyov, M.Y., Werner, F (1997). A polynomial approximation scheme for problem $\mathrm{F} 2 / \mathrm{r}_{\mathrm{j}} / \mathrm{C}_{\max }$. Operations Research Letters 20, 75-79.

Lee, C. Y (1997). Minimizing the makespan in the two-machine flowshop scheduling problem with an availability constraint. Operations Research Letters 20, 129-139.

Lee, C. Y (1999). Two-machine flowshop scheduling with availability constraints. European Journal of Operational Research 114, 420-429. 
Lenstra, J.K., Rinnooy Kan, A.H.G., P., Brucker (1977). Complexity of machine scheduling problems", in: Studies in Integer Programming, eds. P.L. Hammer, E.L. Johnson, B.H. Korter and G.L. Nemhauser, Ann. Discrete Math., North-Holland, Amsterdam 1, 343-362.

Ma, Y., Chu, C., Zuo, C (2010). A survey of scheduling with deterministic machine availability constraints. Computers \& Industrial Engineering 58:2, 199-211.

Potts, C.N (1985). Analysis of heuristics for two-machine flow-shop sequencing subject to release dates. Mathematics of Operations Research 10, 576-584.

Ronconi, D. P (2005). A Branch-and-Bound Algorithm to Minimize the Makespan in a Flowshop with Blocking. Annals of Operations Research 138, 53-65.

Röck, H (1980). The three machine-machine no-wait flow shop problem is NP-complete. Journal of ACM 31, 336-345.

Schmidt, G (2000). Scheduling with limited machine availability. European Journal of Operational Research 121, 1-15.

Wang, G., Cheng, T.C.E (2001). Heuristics for two-machine no-wait flowshop scheduling with availability constraints. Information Processing Letters 80, 305-309. 


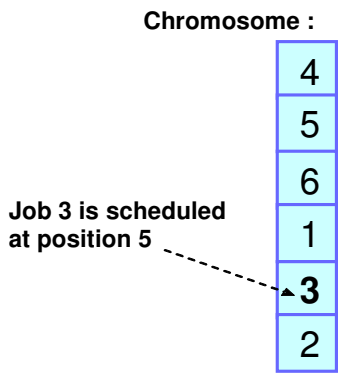

(a) solution representation

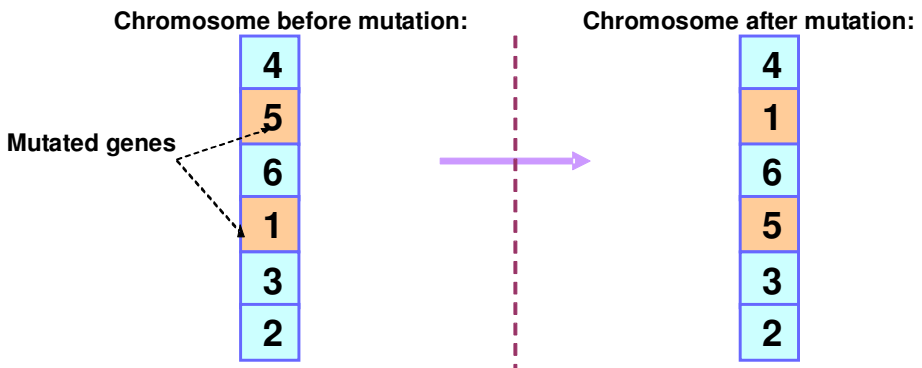

(b) mutation operator

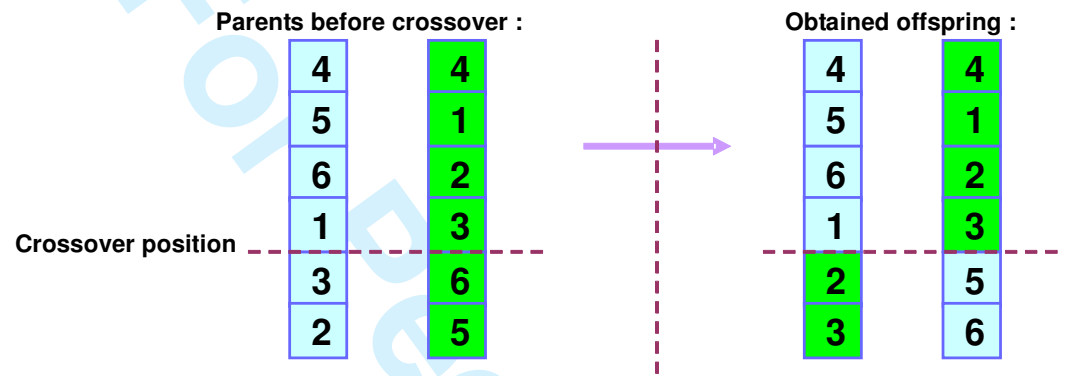

(c) crossover operator

Fig. 1. Representation and operators 
Table1. Group identification

\begin{tabular}{|c|c|c|}
\hline Parameter $R$ & $s_{1}=0.25 \times T$ & $s_{1}=0.5 \times T$ \\
\hline 1 & $\mathbf{1}$ & $\mathbf{2}$ \\
\hline 2 & $\mathbf{3}$ & $\mathbf{4}$ \\
\hline$n$ & $\mathbf{5}$ & $\mathbf{6}$ \\
\hline $2 n$ & $\mathbf{7}$ & $\mathbf{8}$ \\
\hline
\end{tabular}

Table 2. Results of lower and upper bounds for $n=5$

\begin{tabular}{|c|r|r|l|l|r|r|r|r|}
\cline { 2 - 10 } \multicolumn{1}{c|}{} & \multicolumn{4}{c|}{ Average values of LB } & \multicolumn{4}{c|}{ Gap of $U B$ (in \%) } \\
\hline Groups & LB1 & LB2 & LB3 & LB5 & H1 & H2 & GA & GS \\
\hline 1 & 430 & 399 & 411 & $\mathbf{4 5 1}$ & 22 & 11 & 17 & 22 \\
\hline 2 & 464 & 415 & 440 & $\mathbf{4 7 3}$ & 18 & 2 & 12 & 16 \\
\hline 3 & 511 & 420 & 432 & $\mathbf{5 3 1}$ & 3 & 2 & 5 & 6 \\
\hline 4 & 448 & 395 & 414 & $\mathbf{4 7 8}$ & 15 & 7 & 13 & 18 \\
\hline 5 & 563 & 565 & $\mathbf{5 6 7}$ & 563 & 8 & 18 & 17 & 11 \\
\hline 6 & 570 & 564 & $\mathbf{5 8 6}$ & 582 & 12 & 16 & 12 & 8 \\
\hline 7 & $\mathbf{7 9 2}$ & $\mathbf{7 9 2}$ & $\mathbf{7 9 2}$ & $\mathbf{7 9 2}$ & 0 & 2 & 9 & 0 \\
\hline 8 & $\mathbf{8 6 8}$ & $\mathbf{8 6 8}$ & $\mathbf{8 6 8}$ & $\mathbf{8 6 8}$ & 4 & 8 & 10 & 1 \\
\hline
\end{tabular}

Table 3. Results of lower and upper bounds for $n=10$

\begin{tabular}{|c|c|c|c|c|r|r|r|r|}
\multicolumn{1}{c|}{} & \multicolumn{4}{c|}{ Average values of LB } & \multicolumn{4}{|c|}{ Gap of UB (in \%) } \\
\hline Groups & LB1 & LB2 & LB3 & LB5 & H1 & H2 & GA & GS \\
\hline 1 & $\mathbf{6 9 3}$ & 651 & 651 & $\mathbf{6 9 3}$ & 27 & 15 & 12 & 30 \\
\hline 2 & 632 & 535 & $\mathbf{6 5 4}$ & 633 & 30 & 18 & 15 & 28 \\
\hline 3 & 708 & 634 & 626 & 713 & 15 & 2 & 9 & 20 \\
\hline 4 & 758 & 548 & 668 & $\mathbf{7 6 1}$ & 15 & 12 & 7 & 18 \\
\hline 5 & $\mathbf{1 0 3 9}$ & 1036 & 1036 & $\mathbf{1 0 3 9}$ & 10 & 24 & 5 & 3 \\
\hline 6 & 1060 & $\mathbf{1 0 7 3}$ & 1072 & 1061 & 5 & 9 & 4 & 3 \\
\hline 7 & $\mathbf{1 8 8 9}$ & 1884 & 1884 & $\mathbf{1 8 8 9}$ & 2 & 4 & 3 & 0 \\
\hline 8 & $\mathbf{1 9 6 3}$ & 1939 & 1939 & $\mathbf{1 9 6 3}$ & 2 & 3 & 3 & 1 \\
\hline
\end{tabular}

Table 4. Results of lower and upper bounds for $n=15$

\begin{tabular}{|c|r|r|r|r|r|r|r|r|}
\cline { 2 - 10 } \multicolumn{1}{c|}{} & \multicolumn{4}{|c|}{ Average values of LB } & \multicolumn{4}{|c|}{ Gap of UB (in \%) } \\
\hline Groups & LB1 & LB2 & LB3 & LB5 & H1 & H2 & GA & GS \\
\hline 1 & $\mathbf{1 0 0 2}$ & 895 & 904 & $\mathbf{1 0 0 2}$ & 28 & 20 & 11 & 21 \\
\hline 2 & $\mathbf{9 6 2}$ & 815 & 938 & $\mathbf{9 6 2}$ & 23 & 6 & 9 & 14 \\
\hline 3 & $\mathbf{9 3 0}$ & 906 & 906 & $\mathbf{9 3 0}$ & 33 & 14 & 14 & 27 \\
\hline 4 & 925 & 665 & 851 & $\mathbf{9 2 7}$ & 25 & 16 & 7 & 22 \\
\hline 5 & $\mathbf{1 5 6 4}$ & 1563 & 1563 & $\mathbf{1 5 6 4}$ & 3 & 9 & 5 & 2 \\
\hline 6 & $\mathbf{1 5 4 2}$ & 1507 & 1507 & $\mathbf{1 5 4 2}$ & 8 & 14 & 4 & 4 \\
\hline 7 & $\mathbf{2 9 6 2}$ & 2948 & 2948 & $\mathbf{2 9 6 2}$ & 6 & 10 & 1 & 1 \\
\hline 8 & $\mathbf{2 8 2 6}$ & 2810 & 2817 & $\mathbf{2 8 2 6}$ & 7 & 15 & 3 & 0 \\
\hline
\end{tabular}


Table 5. Results of lower and upper bounds for $n=20$

\begin{tabular}{|c|c|c|c|c|r|r|r|r|}
\cline { 2 - 10 } \multicolumn{1}{c|}{} & \multicolumn{4}{c|}{ Average values of LB } & \multicolumn{4}{c|}{ Gap of UB (in \%) } \\
\hline Groups & LB1 & LB2 & LB3 & LB5 & H1 & H2 & GA & GS \\
\hline 1 & 1126 & $\mathbf{1 1 4 0}$ & 1128 & 1138 & 20 & 20 & 13 & 32 \\
\hline 2 & $\mathbf{1 1 9 9}$ & 1195 & 1195 & $\mathbf{1 1 9 9}$ & 8 & 16 & 13 & 30 \\
\hline 3 & 1221 & 1109 & 1099 & $\mathbf{1 2 3 1}$ & 13 & 18 & 8 & 27 \\
\hline 4 & 1244 & 1154 & 1154 & $\mathbf{1 2 5 0}$ & 12 & 19 & 12 & 22 \\
\hline 5 & $\mathbf{2 0 3 4}$ & $\mathbf{2 0 3 4}$ & 2029 & $\mathbf{2 0 3 4}$ & 0 & 6 & 3 & 0 \\
\hline 6 & 2060 & $\mathbf{2 0 6 8}$ & $\mathbf{2 0 6 8}$ & 2060 & 2 & 6 & 5 & 1 \\
\hline 7 & $\mathbf{4 0 2 2}$ & $\mathbf{4 0 2 2}$ & $\mathbf{4 0 2 2}$ & $\mathbf{4 0 2 2}$ & 2 & 4 & 2 & 0 \\
\hline 8 & $\mathbf{4 0 1 6}$ & 4015 & 4015 & $\mathbf{4 0 1 6}$ & 1 & 2 & 2 & 1 \\
\hline
\end{tabular}

Table 6. Summary of computational times of LB and UB (in seconds)

\begin{tabular}{|c|r|r|r|r|r|r|c|c|}
\hline$n$ & $L B 1$ & $L B 2$ & $L B 3$ & $L B 5$ & $H 1$ & $H 2$ & $G A$ & $G S$ \\
\hline 5 & 0 & 0 & 0 & 0 & 0 & 0 & 0,15 & 0,02 \\
\hline 10 & 0 & 0 & 0 & 0 & 0 & 0 & 0,15 & 0,02 \\
\hline 15 & 0 & 0 & 0 & 2 & 0 & 0 & 0,31 & 0,05 \\
\hline 20 & 0 & 0 & 0 & 120 & 0 & 0 & 0,31 & 0,05 \\
\hline
\end{tabular}

Table 7. $B \& B$ performance for $n=5$

\begin{tabular}{|c|r|r|r|r|r|r|r|}
\hline Groups & \multicolumn{1}{|c|}{$U$ Copt } & CPU & $N_{d}$ & $N_{e l}$ & $N_{e x}$ & Gap \\
\hline 1 & 453 & 443 & 0 & 3 & 12 & 33 & 2 \\
\hline 2 & 484 & 467 & 0 & 0 & 23 & 45 & 3 \\
\hline 3 & 520 & 489 & 0 & 6 & 7 & 25 & 6 \\
\hline 4 & 594 & 562 & 0 & 1 & 19 & 33 & 6 \\
\hline 5 & 592 & 553 & 0 & 7 & 10 & 15 & 7 \\
\hline 6 & 644 & 628 & 0 & 5 & 10 & 19 & 2 \\
\hline 7 & 906 & 902 & 0 & 2 & 5 & 7 & 0 \\
\hline 8 & 1009 & 1009 & 0 & 2 & 4 & 3 & 0 \\
\hline
\end{tabular}

Table 8. $B \& B$ performance for $n=10$

\begin{tabular}{|c|r|r|r|r|r|r|r|}
\hline Groups & UB & Copt & CPU & $N_{d}$ & $N_{e l}$ & $N_{e x}$ & Gap \\
\hline 1 & 748 & 708 & 0 & 4 & 146217 & 134475 & 6 \\
\hline 2 & 749 & 713 & 1 & 0 & 287549 & 143090 & 5 \\
\hline 3 & 766 & 736 & 0 & 6 & 131878 & 147749 & 4 \\
\hline 4 & 780 & 745 & 0 & 2 & 95430 & 42601 & 5 \\
\hline 5 & 1094 & 1084 & 0 & 10 & 2220 & 1760 & 1 \\
\hline 6 & 1052 & 1034 & 0 & 17 & 11246 & 9482 & 2 \\
\hline 7 & 1899 & 1899 & 0 & 3 & 2333 & 1571 & 0 \\
\hline 8 & 1968 & 1957 & 0 & 5 & 4424 & 2518 & 1 \\
\hline
\end{tabular}


Table 9. $B \& B$ performance for $n=15$

\begin{tabular}{|c|c|r|r|r|r|r|r|}
\hline Groups & UB & Copt & CPU & $N_{d}$ & $N_{e l}$ & $N_{e x}$ & Gap \\
\hline 1 & 1045 & 1010 & 1154 & 3 & 247862574 & 316505246 & 3 \\
\hline 2 & 1059 & 1019 & 3483 & 0 & 1623109776 & 495159902 & 4 \\
\hline 3 & 1065 & 1019 & 952 & 9 & 170304340 & 254320785 & 5 \\
\hline 4 & 1024 & 974 & 2247 & 5 & 1039966545 & 404465743 & 5 \\
\hline 5 & 1616 & 1604 & 320 & 14 & 4941954 & 3579316 & 1 \\
\hline 6 & 1611 & 1550 & 3600 & 24 & 24620459 & 16281991 & 4 \\
\hline 7 & 2937 & 2935 & 180 & 6 & 4771824 & 2984638 & 0 \\
\hline 8 & 2925 & 2920 & 1600 & 17 & 91077335 & 130196286 & 0 \\
\hline
\end{tabular}

Table 10. $B \& B$ performance for $n=20$

\begin{tabular}{|c|r|r|r|r|r|r|r|r|}
\hline Groups & UB & Copt & $C P U$ & $N_{d}$ & $N_{e l}$ & \multicolumn{1}{|c|}{$N_{e x}$} & Gap \\
\hline $1,2,3,4$ & \multicolumn{6}{|c|}{ The B\&B failed to improve the upper bound before the time limit } \\
\hline 5 & 2089 & 2065 & 1356 & 18 & 10440088 & 16649816 & 1 \\
\hline 6 & 2225 & 2145 & 7200 & 57 & 218131733 & 192697602 & 4 \\
\hline 7 & 3899 & 3887 & 5360 & 12 & 138114617 & 58012023 & 0 \\
\hline 8 & 3810 & 3809 & 6200 & 5 & 152033322 & 232010045 & 0 \\
\hline
\end{tabular}

Table 11. Performance of algorithms $\mathrm{H1}, \mathrm{H} 2, \mathrm{GA}$ and $\mathrm{GS}$ for $\mathrm{n}=30$ to 50

\begin{tabular}{|l|l|l|l|l|l|r|r|r|r|r|}
\hline$n$ & $L B$ & $G A$ & $G S$ & $H 1$ & $H 2$ & $G_{-} H 1$ & $G_{-} H 2$ & $G_{-} G A$ & $G_{-} G S$ & $t \_G S$ \\
\hline 30 & 3087 & 3245 & $\mathbf{3 1 0 4}$ & 3195 & 3403 & $3 \%$ & $10 \%$ & $5 \%$ & $\mathbf{1 \%}$ & 0,1 \\
\hline 40 & 4042 & 4318 & $\mathbf{4 0 7 7}$ & 4154 & 4441 & $3 \%$ & $10 \%$ & $7 \%$ & $\mathbf{1 \%}$ & 0,3 \\
\hline 50 & 4971 & 5524 & $\mathbf{4 9 9 9}$ & 5189 & 5521 & $4 \%$ & $11 \%$ & $11 \%$ & $\mathbf{1 \%}$ & 0,6 \\
\hline
\end{tabular}

